\title{
Potential of zero charge as a sensitive probe for the titration of ionizable self-assembled monolayers
}

\author{
Pablo Ramírez ${ }^{\mathrm{a}}$, Adrian Granero ${ }^{\mathrm{b}}$, Rafael Andreu ${ }^{\mathrm{a}, *}$, Angel Cuesta ${ }^{\mathrm{c}}$, Willem H. Mulder ${ }^{\mathrm{d}}$, \\ Juan José Calvente ${ }^{\mathrm{a}, *}$ \\ a Departamento de Química Física, Universidad de Sevilla, c/ Prof. Garcia Gonzalez s/n, 41012 Sevilla, Spain \\ ${ }^{\mathrm{b}}$ Departamento de Química, Universidad Nacional de Río Cuarto, Argentina \\ 'Instituto de Química Física "Rocasolano", CSIC, 28006 Madrid, Spain \\ ${ }^{\mathrm{d}}$ Department of Chemistry, University of the West Indies, Mona Campus, Kingston 7, Jamaica
}

\section{A R T I C L E I N F O}

\section{Article history:}

Received 10 July 2008

Received in revised form 4 August 2008

Accepted 6 August 2008

Available online 11 August 2008

\section{Keywords:}

Potential of zero charge

Capacitance

Thiol monolayer

Surface dipole

Surface $\mathrm{p} K_{\mathrm{a}}$

Carboxylic acid

\begin{abstract}
A B S T R A C T
Potential of zero charge and interfacial capacitance values of $\omega$-mercaptoalkanoic acid monolayers deposited on $\mathrm{Au}(111)$ are derived from immersion current transients, and are applied to the analysis of their acid properties. Potential of zero charge and capacitance titration curves extend over six $\mathrm{pH}$ units, and exhibit apparent $\mathrm{pK}_{1 / 2}$ values that vary with electrolyte concentration. Electrostatic adsorption of protons at the monolayer-solution boundary provides a quantitative explanation of these observations, and leads to a diffuse layer corrected $\mathrm{p} K_{\mathrm{a}}$ value of 4.3 , which is independent of the thiol chain-length.
\end{abstract}

(C) 2008 Elsevier B.V. All rights reserved.

\section{Introduction}

Self-assembly of functionalized alkanethiols monolayers on metal substrates offers a convenient route to tailor surface properties from predesigned molecular building blocks [1]. Among these properties, the surface potential generated by the organic monolayer is a direct consequence of the dipolar alignment and degree of ionization of its molecules, and it plays a central role in the development of optoelectronic devices [2]. Within an electrochemical environment, where the monolayer is sandwiched between a metal surface and an electrolyte solution, the potential of zero charge (pzc) contains the same information on the electrostatic properties of the monolayer as the surface potential [3]. Control of the pzc can be used to either favour or hinder a given adsorption [4] or charge-transfer process [5] at the electrode surface.

Self-assembled monolayers of $\omega$-mercaptoalkanoic acids constitute useful platforms for electrostatic and covalent immobilization of biomolecules on electrodes [6]. Regardless of their extensive use, there remains some controversy in literature regarding the $\mathrm{p} K_{\mathrm{a}}$ value of surface-anchored carboxylic groups, with

\footnotetext{
* Corresponding authors. Tel.: +34 954557177; fax: +34 954557174.

E-mail addresses: fondacab@us.es (R. Andreu), pacheco@us.es (J.J. Calvente).
}

reported values ranging from 5 up to 10 [7-15]. Potential of zero charge measurements are expected to be well suited to monitor the degree of ionization of surface-anchored groups, since they probe the overall potential drop across the interfacial region, whereas alternative approaches such as contact force [8] or electrokinetic [16] measurements sample part of the diffuse layer only.

We have recently proposed a new method for the determination of the potential of zero charge of gold electrodes modified with thiol monolayers [17]. This method makes use of a vapour deposition protocol, to prevent adsorption of ions and solvent molecules during the self-assembly process, and of the immersion technique [18] to measure the free charge on the metal at a given potential. Potential of zero charge $\left(E_{\mathrm{pzc}}\right)$ and interfacial capacitance $\left(C_{\mathrm{dl}}\right)$ values are then derived from plots of the measured charge as a function of the applied potential. Compared to previous methods, this novel approach was shown to provide more accurate results and to be applicable to any monolayer, irrespective of its degree of hydrophilicity. In this communication we present for the first time potential of zero charge and capacitance titration curves for 3-mercaptopropionic (MPA), 6-mercaptohexanoic (MHA) and 11mercaptoundecanoic (MUA) monolayers deposited on $\mathrm{Au}(111)$, extending from $\mathrm{pH} \sim 2$ to $\sim 13$, and show how the observed behaviour up to $\mathrm{pH} \sim 10$ can be explained in terms of the electrostatic adsorption of protons at the negatively charged organic surface. 


\section{Experimental}

3-Mercaptopropionic acid (MPA), 6-mercaptohexanoic acid (MHA) and 11-mercaptoundecanoic acid (MUA) were purchased from Aldrich. Sodium hydroxide $(\mathrm{NaOH})$, phosphoric acid $\left(\mathrm{H}_{3} \mathrm{PO}_{4}\right)$ and sodium perchlorate $\left(\mathrm{NaClO}_{4}\right)$ were purchased from Fluka. Chemical reagents were used as received. Water was purified with a Millipore Milli-Q system. Thiol monolayers were formed just before the measurement of each immersion transient. The freshly flame-annealed gold surface was exposed to a thiol-saturated atmosphere at $75^{\circ} \mathrm{C}$. Measurements were performed at room temperature, $23 \pm 2{ }^{\circ} \mathrm{C}$. Electrolyte solutions were deaerated with a presaturated argon stream prior to the measurements. Chronoamperometric measurements were carried out with an Autolab PGSTAT30 (Eco Chemie). All potential values are referred to the $\mathrm{Ag}|\mathrm{AgCl}| \mathrm{KCl}$ (sat.) electrode. Further experimental details are described in ref. [17].

\section{Results and discussion}

Fig. 1 depicts the variation of $E_{\mathrm{pzc}}$ and $C_{\mathrm{dl}}$ with solution $\mathrm{pH}$ for a MUA monolayer in contact with $1 \mathrm{M}$ and $0.2 \mathrm{M} \mathrm{NaClO}_{4}$ solutions. As the solution $\mathrm{pH}$ is changed from 2 to 13 , a negative shift of $\sim$ $0.4 \mathrm{~V}$ in the $E_{\mathrm{pzc}}$ value and an increase of $C_{\mathrm{dl}}$ by ca. $50 \%$ are observed. Two steps of $\sim 0.2 \mathrm{~V}$ are evident in the $E_{\mathrm{pzc}}$ titration curves. The first step extends from pH 3 to 9 and parallels the variation of $C_{\mathrm{dl}}$, whereas the second step is rather abrupt and has no counter-

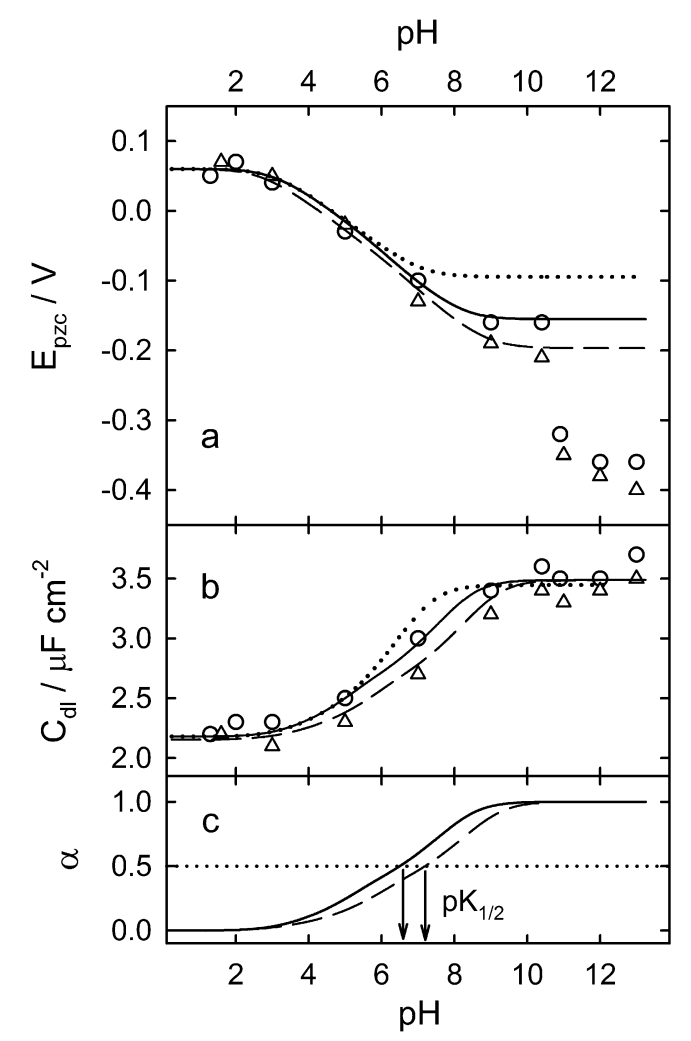

Fig. 1. (a) $E_{\mathrm{pzc}}$ and (b) $C_{\mathrm{dl}}$ titration curves of the MUA monolayer in the presence of (O) $1 \mathrm{M} \mathrm{NaClO}_{4}+10 \mathrm{mM}$ sodium phosphate buffer and $(\triangle) 0.2 \mathrm{M} \mathrm{NaClO}_{4}+5 \mathrm{mM}$ sodium phosphate buffer solutions. Solid and dashed lines are theoretical fits to Eqs. (1) and (3) for $E_{\mathrm{pzc}}$, and to Eqs. (2) and (3) for $C_{\mathrm{dl}}$, taking into account dielectric saturation in the diffuse layer. Dotted lines correspond to $\Delta \phi_{\text {diff }}$ computed from classical Gouy-Chapman theory. (c) Degree of dissociation of the carboxylic groups as a function of solution $\mathrm{pH}$, the arrows indicate the location of the apparent $\mathrm{pK}_{1 / 2}$ values for the two electrolyte concentrations. part in the $C_{\mathrm{dl}}$ titration curve. These differences strongly suggest a different physical origin for the two $E_{\mathrm{pzc}}$ steps. Both the first $E_{\mathrm{pzc}}$ step and the $C_{\mathrm{dl}}$ titration curve can be accounted in terms of a $\mathrm{pH}$ induced dissociation of the surface carboxylic groups, while the second $E_{\mathrm{pzc}}$ step is more likely to originate from the adsorption of hydroxide anions, presumably on the cationic layer that compensates the monolayer charge. A similar behaviour is displayed by the $E_{\mathrm{pzc}}$ and $C_{\mathrm{dl}}$ titration curves of MPA and MHA monolayers, as illustrated in Fig. 2.

In order to attempt a quantitative description of these titration curves, the contributions of the organic monolayer and the diffuse layer to the $E_{\mathrm{pzc}}$ and $C_{\mathrm{dl}}^{-1}$ values are considered to be additive. By further assuming that the potential drop across the thiol monolayer $\left(\Delta \chi_{\text {mon }}\right)$ varies linearly with the degree of dissociation $(\alpha)$ of the carboxylic groups, the pzc value can be expressed as

$E_{\mathrm{pzc}}(\alpha)=E_{\mathrm{pzc}}^{\mathrm{ac}}+\Delta \Delta \chi_{\mathrm{mon}} \cdot \alpha+\Delta \phi_{\mathrm{diff}}(\alpha)$

where $E_{\mathrm{pzc}}^{\mathrm{ac}}$ is the acid limit of $E_{\mathrm{pzc}}, \Delta \Delta \chi_{\mathrm{mon}}$ is the change in surface potential across the monolayer at complete dissociation of the carboxy groups and $\Delta \phi_{\text {diff }}$ is the potential drop across the diffuse layer. Analogously, the interfacial capacitance at the pzc can be expressed as

$C_{\mathrm{dl}}^{-1}(\alpha)=\left(C_{\mathrm{dl}}^{\mathrm{ac}}+\Delta C_{\mathrm{mon}} \cdot \alpha\right)^{-1}+C_{\mathrm{diff}, \text { tot }}^{-1}(\alpha)$

where $C_{\mathrm{dl}}^{\mathrm{ac}}$ is the low $\mathrm{pH}$ limit of $C_{\mathrm{dl}}, \Delta C_{\mathrm{mon}}$ is the change in the monolayer capacitance due to the full ionization of the carboxy groups, and $C_{\text {diff,tot }}(\alpha)$ is the total diffuse layer capacitance, which includes a contribution due to the dissociation process itself [19]. It should be noted that for the relatively high electrolyte concentrations used in this work, $C_{\mathrm{dl}}$ is very sensitive to dielectric changes that occur in the thiol monolayer, but does not contain information

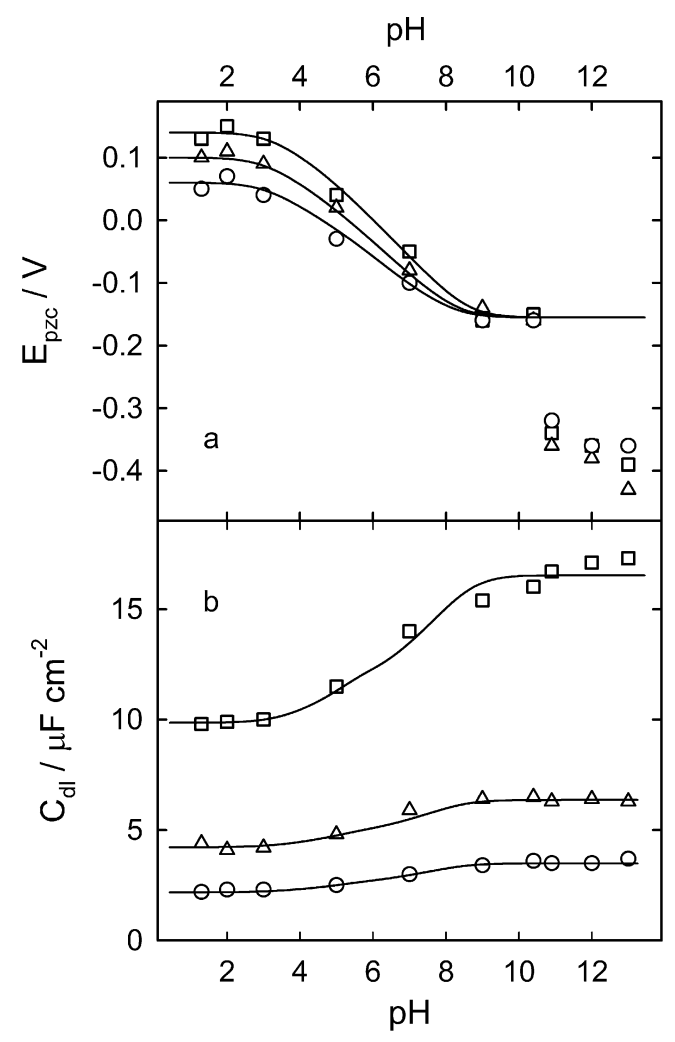

Fig. 2. (a) $E_{\mathrm{pzc}}$ and (b) $C_{\mathrm{dl}}$ titration curves of the (O) MUA, ( $\left.\Delta\right)$ MHA and ( $\square$ ) MPA monolayers in the presence of $1 \mathrm{M} \mathrm{NaClO}_{4}+10 \mathrm{mM}$ sodium phosphate buffer solution. Lines as in Fig. 1. 
Table 1

Parameter values describing the $E_{\mathrm{pzc}}$ and $C_{\mathrm{dl}}$ titration curves

\begin{tabular}{llcll}
\hline & $E_{\mathrm{pzc}}^{\mathrm{ac}} / \mathrm{V}$ & $C_{\mathrm{dl}}^{\mathrm{ac}} / \mu \mathrm{F} \mathrm{cm}^{-2}$ & $\Delta \Delta \chi_{\mathrm{mon}} / V$ & $\Delta C_{\mathrm{mon}} / \mu \mathrm{F} \mathrm{cm}^{-2}$ \\
\hline MUA (1 M) & 0.06 & 2.2 & -0.02 & 1.3 \\
MUA (0.2 M) & 0.06 & 2.2 & -0.02 & 1.3 \\
MHA & 0.10 & 4.3 & -0.06 & 2.1 \\
MPA & 0.14 & 10.3 & -0.10 & 6.5 \\
\hline
\end{tabular}

on the diffuse layer, since $C_{\text {diff,tot }} \gg C_{\mathrm{dl}}^{\mathrm{ac}}+\Delta C_{\text {mon }}$. Moreover, protons are electrostatically attracted towards the negatively charged monolayer, so that their surface concentration differs from the bulk value, and the equilibrium condition leads to [19]

$\mathrm{pH}=\mathrm{p} K_{1 / 2}+\log \left(\frac{\alpha}{1-\alpha}\right)=\mathrm{p} K_{a}-\frac{\mathrm{F} \Delta \phi_{\text {diff }}}{2.303 \mathrm{RT}}+\log \left(\frac{\alpha}{1-\alpha}\right)$

where $K_{a}$ is the acidity constant of the surface-anchored carboxy groups, and $K_{1 / 2}$ is its apparent value obtained by neglecting diffuse layer effects. It may be worth mentioning that the electrochemical titration of these $\omega$-mercaptoalkanoic acid monolayers is expected to differ markedly from those reported for metal oxide adlayers due to the absence of charge exchange processes between the adsorbed film and the electrode [20], and to the availability of a single protonation equilibrium [21], so that the thiol monolayer charge density can only be either negative or zero.

As stated before, Fig. 1 illustrates the influence of electrolyte concentration on the $E_{\mathrm{pzc}}$ and $C_{\mathrm{dl}}$ titration curves of a MUA monolayer. The $\Delta E_{\mathrm{pzc}}$ variation associated with the first step in Fig. 1a is significantly stronger than the $\Delta \phi_{\text {diff }}$ potential drop estimated from the classical Gouy-Chapman theory for a fully ionized monolayer (see the dotted line), but interestingly $\Delta E_{\mathrm{pzc}}$ is quite close to the value computed when dielectric saturation effects are incorporated into the electrostatic description of the diffuse layer. Dielectric saturation effects have been invoked previously by Smalley et al. [12] to explain the ILIT response of $\omega$-mercaptoalkanoic acid monolayers as a function of the solution $\mathrm{pH}$, and they are an inevitable consequence of the high charge density $\left(\sim 80 \mu \mathrm{C} \mathrm{cm}^{-2}\right)$ that prevails at a fully ionized monolayer. Therefore, dielectric saturation effects were incorporated (as described in Ref. [12]) in the $\Delta \phi_{\text {diff }}$ values used to fit the $E_{\mathrm{pzc}}$ and $C_{\mathrm{dl}}$ titration curves. The remaining parameters used for these fits are listed in Table 1.

The fact that the fit parameters are independent of electrolyte concentration indicates that the $E_{\mathrm{pzc}}$ and $C_{\mathrm{dl}}$ changes observed in Fig. 1, in the presence of $1 \mathrm{M}$ (solid lines) and $0.2 \mathrm{M}$ (broken lines) $\mathrm{NaClO}_{4}$ solutions, can be quantitatively accounted for by the calculated differences in $\Delta \phi_{\text {diff }}$ only. This diffuse layer potential drop affects both $E_{\mathrm{pzc}}$ and the proton surface concentration in Fig. 1a and only the proton surface concentration in Fig. 1b. It is interesting to note that the apparent $\mathrm{p} K_{1 / 2}$ value varies from 6.5 ( $1 \mathrm{M}$ solution) to $7.2\left(0.2 \mathrm{M}\right.$ solution), whereas the corrected value $\mathrm{p} K_{\mathrm{a}}$ remains independent of the electrolyte concentration and equal to 4.3 , which is close to the $\mathrm{p} K_{\mathrm{a}}$ value of carboxylic groups in solution. On the other hand, a systematic trend in the fit parameter values with the thiol chain-length is obvious from Table 1 . In the low $\mathrm{pH}$ limit, the $E_{\mathrm{pzc}}$ value increases upon decreasing the hydrocarbon chain-length, as expected from the molecular dipole contribution to $\Delta \chi_{\text {mon }}$ [17], thus confirming the presence of monolayers that are well-ordered in the direction perpendicular to the electrode surface. However, this specific contribution to $\Delta \chi_{\text {mon }}$ is gradually lost as the monolayer becomes more extensively ionized and, finally, a common basic limit of $-0.16 \mathrm{~V}$ is reached for the three monolayers. This loss of molecular order most likely involves only the outer part of the monolayer, since the capacitance values in basic solutions (though higher than in acidic solutions) still retain typical values of densely packed organic monolayers. A single value of $\mathrm{p} K_{\mathrm{a}}=4.3$ was found to reproduce the complete set of $E_{\mathrm{pzc}}$ and $C_{\mathrm{dl}}$ titration curves displayed in Fig. 2, thereby indicating that the acid strength of the terminal carboxylic group is independent of the thiol chain-length.

\section{Conclusions}

Potential of zero charge measurements have been shown to provide useful information on surface ionization process, and it is easy to envisage their application to a variety of processes (such as surface redox or complex formation) that occur on a sufficiently short time scale $(<1 \mathrm{~s})$ and that give rise to a significant change in the surface dipole. Our results illustrate the importance of the surface concentration of protons, rather than its bulk value, as the variable that determines the extent of ionization under well defined electrostatic conditions. The lack of an adequate control of either the local proton concentration, or the electric field experienced by the acid groups, are likely to be the source of some of the discrepancies between the surface $\mathrm{p} K_{\mathrm{a}}$ values reported in litereature.

\section{Acknowledgment}

J.J. Calvente and R. Andreu acknowledge financial support from the DGICYT under grant CTQ 2005-01184 and from the Junta de Andalucía under grant FQM02492.

\section{References}

[1] J.C. Love, L.A. Estroff, J.K. Kriebel, R.G. Nuzzo, G.M. Whitesides, Chem. Rev. 105 (2005) 1103

[2] B. de Boer, A. Hadipour, M.M. Mandoc, T. van Woudenbergh, P.W. Blom, Adv. Mater. 17 (2005) 621.

[3] W.R. Fawcett, Liquids Solutions and Interfaces, Oxford University Press, New York, 2004.

[4] B.A. Kuznetsov, N.A. Byzova, G.P. Shumakovich, J. Electroanal. Chem. 371 (1994) 85.

[5] C.P. Smith, H.S. White, Anal. Chem. 64 (1992) 2398

[6] H. Yue, D.H. Waldeck, J. Petrovic, R.A. Clark, J. Phys. Chem. B 110 (2006) 5062.

[7] C.M. Leopold, J. Black, E.F. Bowden, Langmuir 18 (2002) 978.

[8] D.M. Vezenov, A. Noy, L.F. Rozsnyai, C.M. Lieber, J. Am. Chem. Soc. 119 (1997) 2006.

[9] K.P. Fears, S.E. Creager, R.A. Latour, Langmuir 24 (2008) 837.

[10] M. Tominaga, S. Maetsu, A. Kubo, I. Taniguchi, J. Electroanal. Chem. 603 (2007) 203.

[11] K. Sugihara, K. Shimazu, K. Uosaki, Langmuir 16 (2000) 7101.

[12] J.F. Smalley, K. Chalfant, S.W. Feldberg, T.M. Nahir, E.F. Bowden, J. Phys. Chem. B 103 (1999) 1676.

[13] I. Burgess, B. Seivewright, B.R. Lennox, Langmuir 22 (2006) 4420

[14] T. Kakiuchi, M. Iida, S. Imabayashi, K. Niki, Langmuir 16 (2000) 5397.

[15] S.E. Creager, J. Clarke, Langmuir 10 (1994) 3675.

[16] R. Schweiss, P.B. Welzel, C. Werner, W. Knoll, Colloid Surf. A 195 (2001) 97.

[17] P. Ramírez, R. Andreu, A. Cuesta, C.J. Calzado, J.J. Calvente, Anal. Chem. 79 (2007) 6473.

[18] A. Cuesta, Surf. Sci. 572 (2004) 11.

[19] C.P. Smith, H.S. White, Langmuir 9 (1993) 1.

[20] O.A. Petrii, Electrochim. Acta 41 (1996) 2307.

[21] J. Duval, J. Lyklema, J.M. Kleijn, H.P. van Leeuwen, Langmuir 17 (2001) 7573. 